\title{
Statistical analysis of ground based magnetic field measurements with the field line resonance detector
}

\author{
F. Plaschke ${ }^{1}$, K.-H. Glassmeier ${ }^{1}$, O. D. Constantinescu ${ }^{1}$, I. R. Mann ${ }^{2}$, D. K. Milling ${ }^{2}$, U. Motschmann ${ }^{3}$, and I. J. Rae ${ }^{2}$ \\ ${ }^{1}$ Institut für Geophysik und extraterrestrische Physik, TU Braunschweig, Braunschweig, Germany \\ ${ }^{2}$ Department of Physics, University of Alberta, Edmonton, Canada \\ ${ }^{3}$ Institut für Theoretische Physik, TU Braunschweig, Braunschweig, Germany
}

Received: 21 April 2008 - Revised: 12 September 2008 - Accepted: 10 October 2008 - Published: 10 November 2008

\begin{abstract}
In this paper we introduce the field line resonance detector (FLRD), a wave telescope technique which has been specially adapted to estimate the spectral energy density of field line resonance (FLR) phase structures in a superposed wave field. The field line resonance detector is able to detect and correctly characterize several superposed FLR structures of a wave field and therefore constitutes a new and powerful tool in ULF pulsation studies. In our work we derive the technique from the classical wave telescope beamformer and present a statistical analysis of one year of ground based magnetometer data from the Canadian magnetometer network CANOPUS, now known as CARISMA. The statistical analysis shows that the FLRD is capable of detecting and characterizing superposed or hidden FLR structures in most of the detected ULF pulsation events; the one year statistical database is therefore extraordinarily comprehensive. The results of this analysis confirm the results of previous FLR characterizations and furthermore allow a detailed generalized dispersion analysis of FLRs.
\end{abstract}

Keywords. Magnetospheric physics (MHD waves and instabilities; Instruments and techniques) - Space plasma physics (Wave-wave interactions)

\section{Introduction}

For several decades ULF pulsations have been observed using ground based magnetometer measurements. Pioneering work in interpreting these pulsations was first done by Dungey (1954), who found eigensolutions of a dipole magnetosphere assuming spatially constant plasma density. Later Tamao (1965) showed the possibility of excitation of resonant ULF waves within the magnetosphere. Experimental work by Samson et al. (1971) was crucial in determining am-

Correspondence to: F. Plaschke

(f.plaschke@tu-bs.de) plitude and phase structures of the observed pulsations and finally led to the theoretical approaches of Chen and Hasegawa (1974) and Southwood (1974), who developed a theory for field line resonances (FLR) consistent with the observations. Their analytical model describes the coupling of evanescent compressional disturbances generated by a surface wave to localized standing Alfvén waves at a resonance point, where the driving frequency of the surface wave and the local eigenfrequency coincide. The resulting Alfvén mode oscillations are characterized by a $180^{\circ}$ phase shift and a polarization change across the resonance region as well as a local amplitude maximum.

Though several sources of the initial compressional disturbance like solar wind driven closed cavity or open waveguide oscillations in their respective eigenfrequencies (e.g. Allan et al., 1986; Samson et al., 1992; Mann et al., 1999) or Kelvin-Helmholtz instability surface waves on the flanks of the magnetopause (e.g. Southwood, 1974; Fujita et al., 1996; Engebretson et al., 1998; Rae et al., 2005) have been proposed, the spatial amplitude and phase structures of ground based magnetic field measurements during FLR events are commonly accepted due to abundant experimental evidence (e.g. Samson et al., 1971; Green, 1981; Glassmeier et al., 1999): Toroidal mode standing Alfvén wave oscillations generate dominant disturbances in the latitudinal $H$ component of the ground magnetic field (see Hughes, 1974) with an $180^{\circ}$ phase shift in meridional direction and a small azimuthal wavenumber (large wavelength in zonal direction). Additionally, these phase structures have been observed to move predominantly tailward in the morning and afternoon local time sectors demonstrating mainly poleward phase front motion at high latitude resonance regions (e.g. Glassmeier et al., 1984; Fenrich et al., 1995).

The determination of the parameters which characterize a FLR event (namely the latitudinal phase shift $\alpha$, the width of the phase shift area on the ground $p$, the meridional location of the resonating field line shell $x_{0}$ and the azimuthal

Published by Copernicus Publications on behalf of the European Geosciences Union. 
wavenumber $m$ ) can be achieved comparing phase differences of timeseries of $H$ component magnetic field disturbances from either meridionally or zonally adjacent stations of a ground magnetometer array. These phases relative to a reference signal can be computed for each station's signal by standard Fourier transform or by complex demodulation (Beamish et al., 1979). For the successful application of these methods large pulsation amplitudes at a number of stations and mainly time independent phase differences between them are required, as there is no array with sufficiently high spatial resolution to perform a Fourier transform of the given phase structure into an adapted wavevector space. Hence, their application is restricted to clearly observed single FLR events, as the methods struggle with the correct decomposition of two or more superposed FLR phase structures within a narrow frequency band (e.g. Rae et al., 2007a).

The intention of this work is to introduce an adaptation of the wave telescope technique, in order to relax the strict constraints outlined above. The wave telescope is an array signal processing technique, also known as Capon beamformer, which was introduced by Capon et al. (1967) and Capon (1969) for the analysis of seismic waves. Essentially, this technique is a high resolution estimator for the spectral field energy density of a wave field measured at a sparse array of stations with good temporal resolution. With this method it is possible to decompose a wave field of several superposed plane waves without the need of a spatially dense array of stations. Pillai (1989) proved the wave telescope to be a generalized minimum variance technique. Pinçon and Lefeuvre (1991) expanded the method to include multidimensional (vectorial) observables and Motschmann et al. (1996) further developed it in order to apply it to multipoint satellite measurements (e.g. Glassmeier et al., 2001; Narita et al., 2003; Constantinescu et al., 2007).

In this paper we adapt the wave telescope to the FLR phase structure and call the new technique: field line resonance detector (FLRD). Furthermore we apply this technique to automatically selected ULF magnetic pulsation events of the year 2002 measured at the ground based Canadian magnetometer array CANOPUS (Rostoker et al., 1995), now known as CARISMA (Canadian Array for Realtime Investigations of Magnetic Activity, www.carisma.ca), to benchmark the results.

\section{The Field Line Resonance Detector}

For the derivation of the FLRD we start from the known phase structure of a field line resonance in geomagnetic coordinates. In latitudinal direction it should display a $180^{\circ}$ phase shift, whereas in longitudinal direction the phase changes linearly and the corresponding wavelength is determined by the azimuthal wavenumber $m$. Due to the curvature of the Earth's surface the real wavelength in zonal direction is con- stant only on a given parallel and changes with the latitude thereof:

$m=k_{y} \cdot R_{\mathrm{E}} \cdot \cos \left(\vartheta_{\mathrm{RP}}\right)$

Here $R_{E}$ denotes the radius of the Earth and $\vartheta_{\mathrm{RP}}$ the latitude of the reference parallel.

In order to make $k_{y}$ constant over the whole area of appearance of the FLR on the ground we transform the phase structure from the surface geomagnetical coordinate system to a plane by equirectangular projection. Given an arbitrary reference point (RP) at any position on Earth by longitude and latitude $\left(\varphi_{\mathrm{RP}}, \vartheta_{\mathrm{RP}}\right)$, any other position $\left(\varphi_{s}, \vartheta_{s}\right)$ is transformed in the following manner:

$x_{s}=R_{E} \cdot \frac{2 \pi}{360^{\circ}} \cdot\left(\vartheta_{s}-\vartheta_{\mathrm{RP}}\right)$

$y_{s}=R_{E} \cdot \frac{2 \pi}{360^{\circ}} \cdot \cos \left(\vartheta_{\mathrm{RP}}\right) \cdot\left(\varphi_{s}-\varphi_{\mathrm{RP}}\right)$

$x_{s}$ and $y_{s}$ can now be interpreted as coefficients of basis vectors of a cartesian coordinate system with $x$ and $y$ pointing northward and eastward, respectively. Due to the scaling factors in Eqs. (2) and (3) the coordinate axes coincide in length scale with the meridian and parallel crossing the reference point. As a result of the transform the zonal wavenumber $k_{y}$ measured at that reference parallel applies now to every location in the equirectangular projection and therefore becomes independent of the latitude.

In this cartesian coordinate system an approximative description of the phase structure of a FLR and therefore of the magnetic field variation $b$ is given by:

$$
\begin{aligned}
& b(x, y, t) \\
= & b_{0} \cdot \exp \left[\frac{\left(x-x_{0}\right)^{2}}{2 \sigma_{x}^{2}}\right] \cdot \exp \left[\frac{\left(y-y_{0}\right)^{2}}{2 \sigma_{y}^{2}}\right] \\
\cdot & \cos \left[\frac{\alpha}{2} \arctan \left(\frac{x-x_{0}}{p}\right)+\left(k_{y}\left(y-y_{0}\right)-\omega t\right)+\varphi\right]
\end{aligned}
$$

where $x_{0}$ and $y_{0}$ denote the center of the FLR phase structure on ground, $\sigma_{x}$ and $\sigma_{y}$ are scaling factors of the amplitude distribution and $\alpha$ is the total phase shift in meridional direction, which in theory should be $\pi . b_{0}$ represents here the amplitude of the magnetic field disturbance measured on the ground, $p$ is the parameter which adjusts the latitudinal width of the phase shift region. $\omega$ and $\varphi$ are, as usual, the angular frequency of the pulsation and its initial phase, respectively.

A similar expression has previously been used by Glassmeier (1988) for modelling the amplitude of electric field variations of FLRs. We will now use Eq. (4) to adapt the basis functions of the wave telescope. A detailed derivation of the wave telescope technique has already been done by Motschmann et al. (1996). In order to make differences between both techniques understandable we will repeat some of the computations of the mentioned work and use similar symbols. 
The wave telescope and therefore the field line resonance detector (FLRD) are beamforming techniques, which estimate the spectral field energy density of a particular wave mode. In the original technique the wave modes searched for correspond to plane waves with arbitrary wave vector $\boldsymbol{k}$ and frequency $\omega$. Let $\boldsymbol{b}\left(\boldsymbol{r}_{s}, t\right)$ be a magnetic field pertubation at the position $\boldsymbol{r}_{s}$ and the time $t$ resulting from the superposition of $N$ plane waves with wave vectors $\boldsymbol{k}_{n}$ :

$\boldsymbol{b}\left(\boldsymbol{r}_{s}, t\right)=\sum_{n=1}^{N} \boldsymbol{b}_{n} \cdot e^{i \boldsymbol{k}_{n} \cdot \boldsymbol{r}_{s}} \cdot e^{-i \omega_{n} t}$

As $\boldsymbol{b}\left(\boldsymbol{r}_{s}, t\right)$ can be measured at any station position $\boldsymbol{r}_{s}$ with high temporal resolution, Fourier transformation of the timeseries to frequency domain is possible yielding the known result:

$\boldsymbol{b}\left(\boldsymbol{r}_{s}, \omega\right)=\frac{1}{J} \sum_{j=1}^{J} \boldsymbol{b}\left(\boldsymbol{r}_{s}, t_{j}\right) \cdot e^{i \omega t_{j}}$

where the index $j$ denotes the measured field vector samples. Further transformation to the wave vector domain $\{\boldsymbol{b}(\boldsymbol{k}, \omega)\}$ would not yield a satisfactory result, since the spatial resolution of the phase structure with few stations $(S)$ is unavoidably poor. The spectral energy density $P(\boldsymbol{k}, \omega)$ for the plane wave contribution with wave vector $\boldsymbol{k}$ and frequency $\omega$ therefore cannot be directly computed by the following formulae:

$$
\begin{aligned}
& \mathbf{P}(\boldsymbol{k}, \omega)=\left\langle\boldsymbol{b}(\boldsymbol{k}, \omega) \cdot \boldsymbol{b}^{\dagger}(\boldsymbol{k}, \omega)\right\rangle \\
& P(\boldsymbol{k}, \omega)=\operatorname{tr}[\mathbf{P}(\boldsymbol{k}, \omega)]
\end{aligned}
$$

where the spectral energy density matrix $\mathbf{P}(\boldsymbol{k}, \omega)$ is the expectation value of the dyadic product of the Fourier coefficient vector $\boldsymbol{b}(\boldsymbol{k}, \omega)$. Here the dagger $\left(^{\dagger}\right)$ indicates the Hermitian adjoint of the matrix, that means the transposed complex conjugate of the original matrix.

Having introduced the observation vector $\boldsymbol{B}(\omega)$

$\boldsymbol{B}(\omega)=\left(\begin{array}{c}\boldsymbol{b}\left(\boldsymbol{r}_{1}, \omega\right) \\ \vdots \\ \boldsymbol{b}\left(\boldsymbol{r}_{S}, \omega\right)\end{array}\right)$

which is a known quantity, as well as the phase compensation matrix $\mathbf{H}(\boldsymbol{k})$

$\mathbf{H}(\boldsymbol{k})=\left(\begin{array}{c}\mathbf{I} \cdot e^{i \boldsymbol{k} \cdot \boldsymbol{r}_{1}} \\ \vdots \\ \mathbf{I} \cdot e^{i \boldsymbol{k} \cdot \boldsymbol{r}_{S}}\end{array}\right)$

(I: identity matrix) and the observation matrix $\mathbf{M}(\omega)$, which is the expectation value of the dyadic product of the observation vector $\boldsymbol{B}$,

$\mathbf{M}(\omega)=\left\langle\boldsymbol{B}(\omega) \cdot \boldsymbol{B}^{\dagger}(\omega)\right\rangle$ another expression for the spectral energy density matrix can be found, which is characteristic for the beamforming method introduced by Capon et al. (1967):

$\mathbf{P}(\boldsymbol{k}, \omega)=\left(\mathbf{H}^{\dagger}(\boldsymbol{k}) \cdot \mathbf{M}^{-1}(\omega) \cdot \mathbf{H}(\boldsymbol{k})\right)^{-1}$

This equation is the result of a Lagrangian minimization of the spectral energy density $P$ with the constraint, that the spectral energy corresponding to the wave of wave vector $\boldsymbol{k}$ and frequency $\omega$ is preserved (for further details see Motschmann et al., 1996). The structure of the phase compensation matrix $\mathbf{H}$ is irrelevant for this minimization procedure. However, $\mathbf{H}$ contains the phase structure of the wave, of which the energy density content in $P$ is hold constant. Therefore it is easily possible to adapt the wave telescope technique for the detection of FLRs by simply replacing the phase expressions in Eq. (10) with the phase function, argument of the cosine in Eq. (4):

$$
=\left(\begin{array}{c}
\mathbf{H} \cdot \exp \left[i\left(\alpha \arctan \left(\frac{x_{1}-x_{0}}{p}\right)+k_{y} y_{1}\right)\right] \\
\vdots \\
\mathbf{I} \cdot \exp \left[i\left(\alpha \arctan \left(\frac{x_{S}-x_{0}}{p}\right)+k_{y} y_{S}\right)\right]
\end{array}\right)
$$

Rewriting Eq. (12) yields a spectral energy density function $P$, which is no longer dependent on the wave vector $\boldsymbol{k}$, but on the parameters $\alpha, x_{0}, p$ and $k_{y}$, which characterize the spatial FLR phase structure:

$$
\begin{aligned}
& P\left(\alpha, x_{0}, p, k_{y}, \omega\right) \\
= & \operatorname{tr}\left[\left(\mathbf{H}^{\dagger}\left(\alpha, x_{0}, p, k_{y}\right) \cdot \mathbf{M}^{-1}(\omega) \cdot \mathbf{H}\left(\alpha, x_{0}, p, k_{y}\right)\right)^{-1}\right]
\end{aligned}
$$

Therefore expression (14) becomes the fundamental equation of the field line resonance detector.

It should be noted that mathematically there is a difference between the classical wave telescope and the FLRD: The wave telescope estimates the spectral energy density in the coefficient space of plane wave basis functions $\{\exp (i \boldsymbol{k} \cdot \boldsymbol{r})\}$. The functions used in the phase compensation matrix $\mathbf{H}$ instead do not constitute a set of basis functions in the mathematically strong sense. This means that there may be wave fields which are not representable by linear combination of these functions or (less likely) that linear combination of the functions with two different sets of coefficients may result in the same wave field. Since the FLRD does only select the most likely functions to describe the FLR phase structure, a complete decomposition of the wave field into basis functions (as done by a Fourier tranform) is not seeked for and therefore completeness of the set of functions is not necessary.

The application of the FLRD is similar to the wave telescope technique. A common interval of magnetic field vectors measured at different adjacent ground stations is first selected. This interval has to be divided into several (overlapping) subintervals, which are then Fourier transformed into 
Table 1. The coordinates of the 13 CANOPUS stations in geographic and geomagnetic coordinate systems and in the equirectangular projection of the geomagnetic coordinate system.

\begin{tabular}{lcrrrrrr}
\hline \multirow{2}{*}{ Stations } & \multicolumn{2}{c}{ Geographic } & \multicolumn{2}{c}{ Geomagnetic } & \multicolumn{2}{c}{ Equirect. Proj. } \\
Station & Code & Latitude & Longitude & Latitude & Longitude & $\mathrm{x}[\mathrm{km}]$ & $\mathrm{y}[\mathrm{km}]$ \\
\hline Contwoyto & CONT & $65.8^{\circ}$ & $248.8^{\circ}$ & $72.5^{\circ}$ & $-60.7^{\circ}$ & 617 & -247 \\
Dawson & DAWS & $64.0^{\circ}$ & $220.9^{\circ}$ & $66.2^{\circ}$ & $-89.6^{\circ}$ & -92 & -1502 \\
Eskimo Point & ESKI & $61.1^{\circ}$ & $266.0^{\circ}$ & $70.4^{\circ}$ & $-33.1^{\circ}$ & 373 & 950 \\
Fort Churchill & FCHU & $58.8^{\circ}$ & $265.9^{\circ}$ & $68.1^{\circ}$ & $-31.9^{\circ}$ & 117 & 1004 \\
Fort Simpson & FSIM & $61.8^{\circ}$ & $238.8^{\circ}$ & $67.2^{\circ}$ & $-68.3^{\circ}$ & 23 & -579 \\
Fort Smith & FSMI & $60.0^{\circ}$ & $248.1^{\circ}$ & $67.0^{\circ}$ & $-55.9^{\circ}$ & 5 & -38 \\
Gillam & GILL & $56.4^{\circ}$ & $265.4^{\circ}$ & $65.7^{\circ}$ & $-31.6^{\circ}$ & -150 & 1018 \\
Island Lake & ISLL & $53.9^{\circ}$ & $265.3^{\circ}$ & $63.1^{\circ}$ & $-30.6^{\circ}$ & -426 & 1058 \\
Fort McMurray & MCMU & $56.7^{\circ}$ & $248.8^{\circ}$ & $63.9^{\circ}$ & $-52.7^{\circ}$ & -342 & 99 \\
Pinawa & PINA & $50.2^{\circ}$ & $264.0^{\circ}$ & $59.4^{\circ}$ & $-31.2^{\circ}$ & -843 & 1032 \\
Rabbit Lake & RABB & $58.2^{\circ}$ & $256.3^{\circ}$ & $66.5^{\circ}$ & $-44.3^{\circ}$ & -60 & 465 \\
Rankin Inlet & RANK & $62.8^{\circ}$ & $267.9^{\circ}$ & $72.2^{\circ}$ & $-31.4^{\circ}$ & 581 & 1023 \\
Taloyoak & TALO & $69.5^{\circ}$ & $266.5^{\circ}$ & $78.5^{\circ}$ & $-40.9^{\circ}$ & 1284 & 611 \\
\hline
\end{tabular}

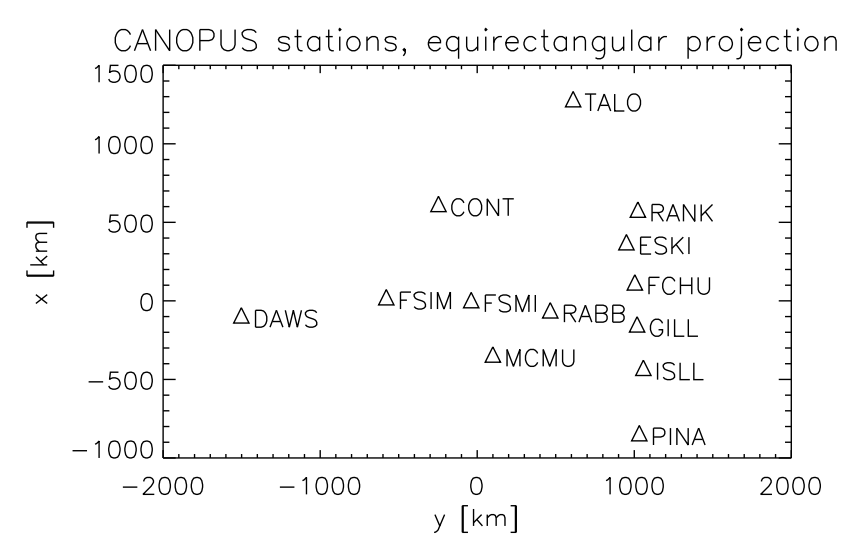

Fig. 1. Positions of the 13 stations of the CANOPUS array in equirectangular projection of the geomagnetic coordinate system. Positions of the geomagnetic North Pole and the reference point (RP) are given in the text.

frequency domain. A prominent frequency $\omega$, pulsations are observed at, is selected and complex observation vectors $\boldsymbol{B}_{i}(\omega)$ are computed for each subinterval indexed with $i$. The observation covariance matrix $\mathbf{M}$ is given by the mean of the observation matrices $\mathbf{M}_{i}$ of the subintervals:

$$
\begin{aligned}
\mathbf{M}_{i}(\omega) & =\boldsymbol{B}_{i}(\omega) \cdot \boldsymbol{B}_{i}^{\dagger}(\omega) \\
\mathbf{M}(\omega) & =\frac{1}{I} \sum_{i=1}^{I} \mathbf{M}_{i}
\end{aligned}
$$

Now a subdomain in the four-dimensional parameter space composed by $\alpha, x_{0}, p$ and $k_{y}$ is chosen and suitably discretized. For each combination of parameters the phase compensation matrix $\mathbf{H}$ is computed according to Eq. (13). Now all the matrices necessary to compute the spectral energy density matrix and thus the spectral energy density $P$ by Eq. (14) are available. This procedure is repeated until $P$ is calculated for all chosen combinations of parameters. The local maxima in four-dimensional parameter space can now be interpreted as FLR phase structure contributions to the wave field measured at the ground stations. In a statistical analysis this further allows to determine dependences between the FLR characterizing parameters providing generalized dispersion relations of FLRs.

\section{Data and event preselection}

For our statistical analysis we used one year, 2002, of ground based magnetic field measurements observed at the Canadian CANOPUS magnetometer array. The array consists of 13 stations covering large part of the Canadian landmass between $50^{\circ}$ and $70^{\circ}$ geographic latitude. Its sensor positions in the geographic and geomagnetic coordinate systems as well as in the equirectangular projection of the geomagnetic coordinate system are shown in Table 1. For the computation of these values a geomagnetic North Pole position of $79.6^{\circ}$ latitude and $-71.7^{\circ}$ longitude has been assumed according to the IGRF-10 dipole field model for 2002 (Maus et al., 2005). A central position at $\varphi_{\mathrm{RP}}=-55^{\circ}$ longitude and $\vartheta_{\mathrm{RP}}=67^{\circ}$ latitude in geomagnetic coordinates has been chosen as the reference point, which corresponds to the position $\left(x_{\mathrm{RP}}=0 \mathrm{~km}, y_{\mathrm{RP}}=0 \mathrm{~km}\right)$ in the equirectangular projection. In this coordinate system the locations of all 13 CANOPUS stations are displayed in Fig. 1.

For the statistical analysis of ground-based data with the FLRD a preselection of low frequency pulsation events has to be performed first. In this preselection process the analysis frequency $\omega$ and the time interval where pulsations are observed must be selected. Vector magnetic field data sampled 
Table 2. Intervals in parameter space, which determine the scanning area for the FLRD application: Shown are minimum and maximum for each parameter and the discretization steps.

\begin{tabular}{lcccc}
\hline Parameter description & Symbol & Minimum & Maximum & Discretization \\
\hline phase shift & $\alpha$ & $140^{\circ}$ & $220^{\circ}$ & $10^{\circ}$ \\
width of phase shift & $p$ & $-700 \mathrm{~km}$ & $700 \mathrm{~km}$ & $50 \mathrm{~km}$ \\
center of phase shift in equirect. proj. & $x_{0}$ & $-1000 \mathrm{~km}$ & $1500 \mathrm{~km}$ & $50 \mathrm{~km}$ \\
corresponding geomag. latitude & $\vartheta_{0}$ & $58.0^{\circ}$ & $80.5^{\circ}$ & $0.45^{\circ}$ \\
zonal wavenumber & $k_{y}$ & $-0.0075 \mathrm{~km}^{-1}$ & $0.0075 \mathrm{~km}^{-1}$ & $1.5 \times 10^{-4} \mathrm{~km}^{-1}$ \\
corresponding azimuthal wavenumber & $m$ & -18.67 & 18.67 & 0.37 \\
\hline
\end{tabular}

at $5 \mathrm{~s}$ intervals was available for large parts of the array throughout the whole year 2002. In order to obtain samples at common supporting points all timeseries were first resampled at a rate of $1 \mathrm{~Hz}$. For further computations all field vectors were transformed to the geomagnetic coordinate system, with $b_{x}$ and $b_{y}$ pointing northward (new $H$ component) and eastward (new $D$ component) respectively. Dynamic power spectra of the northward $H$ component were obtained from $1024 \mathrm{~s}$ intervals shifted by $1 \mathrm{~min}$. This interval length was chosen as a trade-off between frequency and time resultion. Previous to the calculations the linear trends of the $H$ timeseries intervals were removed and a $5 \%$ cosine window was applied to the intervals. The power spectra were computed using classical FFT.

To take account only for long periodic pulsations in the Pc5 frequency range, the low frequency parts of the power spectra (below $25 \mathrm{mHz}$ ) were approximated with and divided by a suitable power law. The decadic logarithm of the resulting values displays the magnitude of power of an oscillatory component over a mean noise value given by the power law fit. If one component is steadily above a certain threshold, it can be considered a peak in the power spectrum. This threshold level has been set to 1 , which means that the power value of one oscillatory component of the spectrum has to be at least one order of magnitude larger than the power law fit to the whole spectrum at that given frequency. If one power component fulfills this criterion for at least 5 consecutive intervals, it is considered a pulsation event at that frequency. The duration of an event is obtained from the number of consecutive maxima for which the criterion is fulfilled.

For each frequency bin all pulsation events found at the 13 stations are then merged such that two or more temporally overlapping events observed at two different stations become one single event covering both events temporally. The resulting database of pulsation events, which is composed of starting times for the pulsations events, their total duration and pulsation frequencies is used for further analysis.

The pulsation intervals are now divided into 32 equidistant subintervals, which are needed for the computation of the estimate of the observation vector in the FLRD method. For each of these subintervals the spectral power density of the $H$ component at every station is recalculated and a mean value at the event frequency per station is computed. Stations are chosen for the subsequent FLRD analysis, whose power value is not below one order of magnitude less than the maximal mean power value registered at one station for the given event. This is necessary, because the FLRD method does not include a fitting of the amplitude distribution and assumes a constant amplitude of the pulsations over the whole measurement area. If stations are chosen for the computations with very low relative power at the analysis frequency, noise will play an important role in the fitting process by the FLRD. Therefore measurements of stations showing low pulsation power have to be dismissed before the analysis.

For the described subinterval distribution and the chosen stations (if more than 3 remain) the FLRD method is applied to the $H$ component of the magnetic field at the given event frequency. The scanning procedure is done for the area given in Table 2 within the parameter domain. The discretization steps are also shown in that table.

Finally all local maxima of the spectral energy density in the parameter domain are searched for. The location of these maxima constitute the result of the statistical FLRD analysis.

\section{Results and discussion}

After the first preselection process 28189 events remained for the FLRD analysis. The result of every FLRD computation is a list of local maxima of the spectral energy density in the scanned area in parameter space. The positions of these maxima in this four-dimensional space provide us with the parameters of the FLR phase structures that contribute to the measured wave field. In order to select only these types of structures which can clearly be attributed to toroidal field line oscillations, we chose a narrow bandwidth for the $\alpha$ parameter (phase shift) around $\pi\left(180^{\circ}\right)$ for the scanning domain and dismissed all but the strongest maxima of each FLRD analysis. Taking into account the events that were dismissed due to the number of stations where the pulsations were observed and the events that did not show any significant contribution of power from waves that are characterized by the parameter combinations of the scanning domain, we could condense 


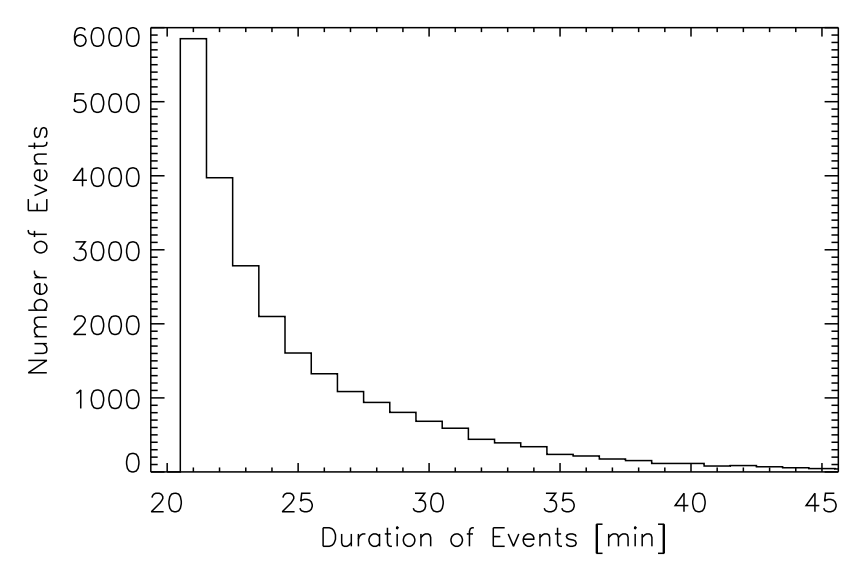

Fig. 2. Distribution of FLR event duration. Low duration events are clearly predominant. Binning used for the histogram: $1 \mathrm{~min}$.

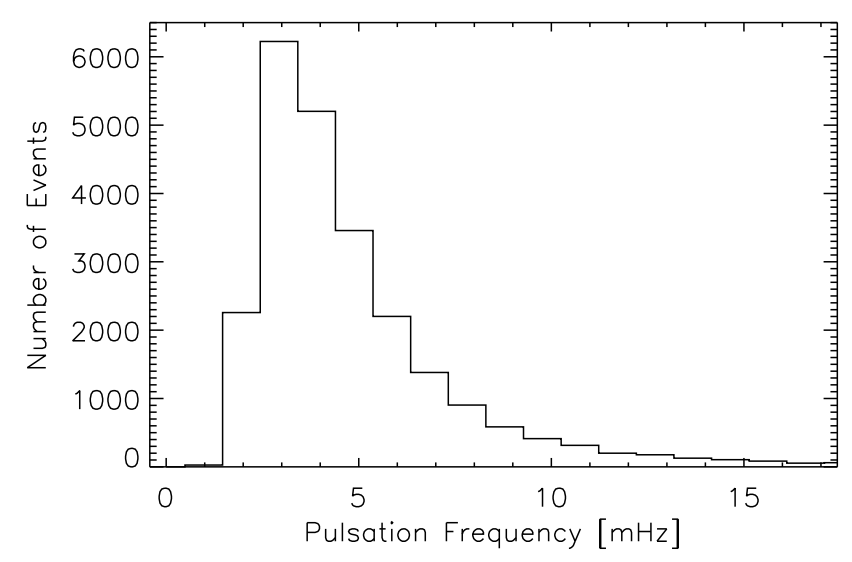

Fig. 3. Distribution of the pulsation frequency of the events analyzed with the FLRD method. $3 \mathrm{mHz}$ oscillations are clearly predominant. Binning used for the histogram: 1/1024 s.

the whole year of magnetic field data to 24645 sets of FLR characterizing parameters.

The duration of the FLR events ranges from 21 min to over one hour. The lower end of this range is the minimum duration possible due to the preselection requirement of 5 consecutive power maxima ( $4 \mathrm{~min}$ or $240 \mathrm{~s}$ ) over the threshold level and the FFT interval length of $1024 \mathrm{~s}$ yielding a total interval length of $1024+240=1264 \mathrm{~s}$ which roughly equals $21 \mathrm{~min}$. The number of events as a function of their duration is shown in Fig. 2. The amplitude of FLRs is known to decrease with time as both the driving mechanism is switched off and it is damped by ionospheric Joule heating (e.g. Greenwald and Walker, 1980; Rae et al., 2007b). Hence, the probability of events fulfilling the preselection criteria also decreases with time. This results in an exponential decay of the occurrence probability of larger continuous high amplitude pulsations as depicted in Fig. 2.

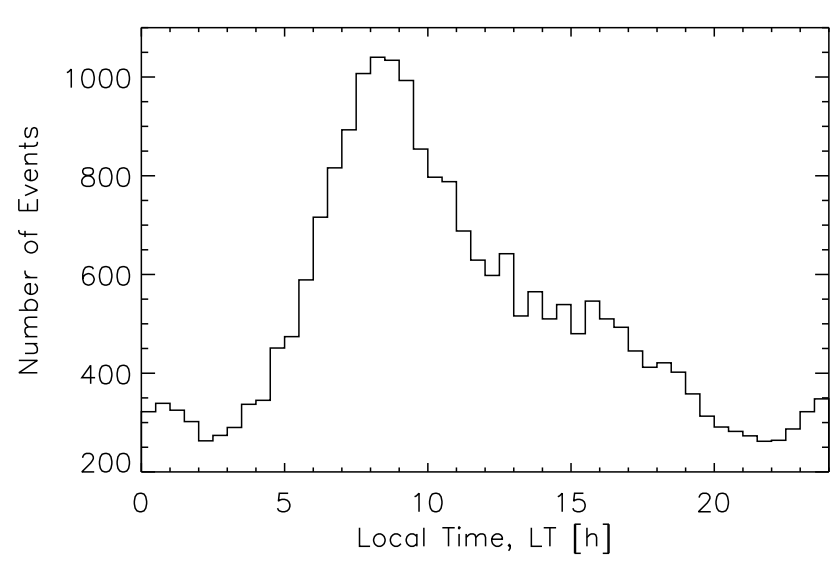

Fig. 4. Distribution of events in local time of the center point of the station distribution involved in the analysis of each event taken at the mean time of appearance of the pulsation. Binning used for the histogram: half an hour.

In Fig. 3 the frequency distribution of the FLR events is shown. It is obvious that pulsation events at a frequency of $3 \mathrm{mHz}$ are predominant with little spreading in frequency. Since the array is located on a relatively narrow latitude band and most stations are located around $67^{\circ}$ geomagnetic latitude, the narrow frequency band is most probably the result of the eigenfrequency distribution of the field line shells whose footpoints are close to the CANOPUS stations. The result is in agreement with the work of Ziesolleck and McDiarmid (1995) and Chisham and Orr (1997) as well, who observed a preferential appearance of Pc5 pulsations at frequencies in the $2 \mathrm{mHz}$ to $5 \mathrm{mHz}$ range.

The distribution of observed events against local time is shown in Fig. 4. The local time of appearance of an event is computed using the mean time of the total analysis interval and the mean longitude (in geographic coordinates) of the station configuration which was used for the FLRD analysis. The distribution of the events with respect to the local time of appearance shows a clear dawn-dusk asymmetry also observed in numerous other studies (e.g. Engebretson et al., 1998; Glassmeier and Stellmacher, 2000; Baker et al., 2003). The results obtained by Engebretson et al. (1998) using a Pc5 index are directly comparable to the presented ones as well, since this index is basically the quotient between power in the Pc5 frequency band and total power over a larger band. In our case the preselection process itself is sensitive only for low frequencies in that band, and whenever a narrow banded pulsation signal occurs with sufficient power with respect to other frequencies, a pulsation event is counted which contributes to the result in Fig. 4.

Another interesting parallel to the study of Engebretson et al. (1998) is the appearance of a periodicity of about 27 days in the occurrence rate of events shown in Fig. 5. In this figure the lower panel shows the distribution of events during 


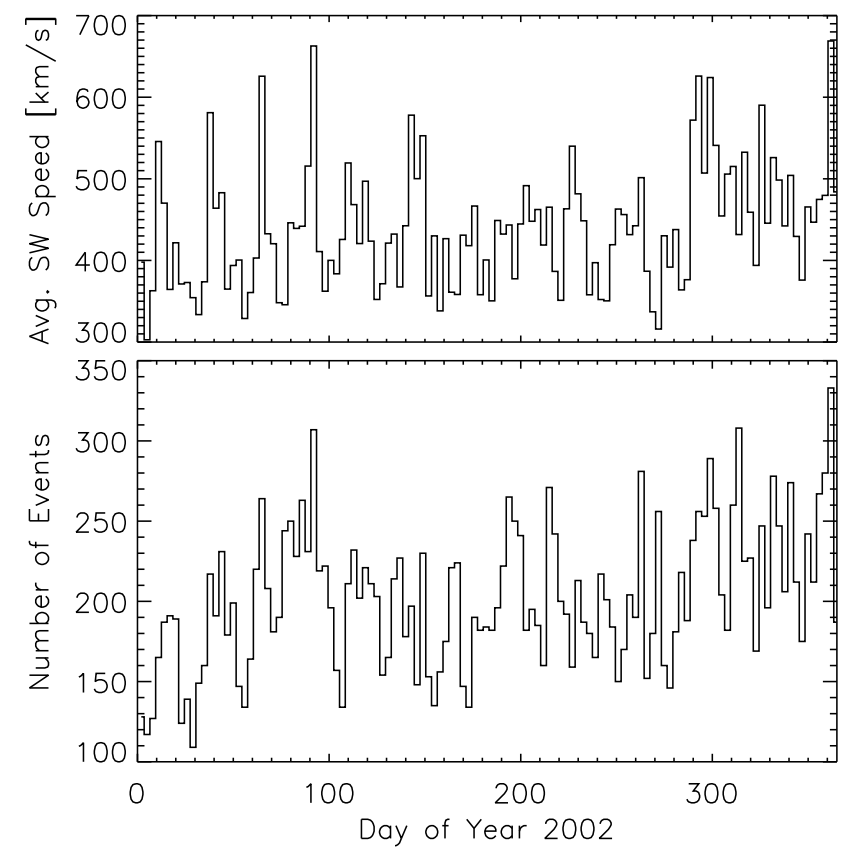

Fig. 5. Lower panel: Occurrence of events during the year 2002. Upper panel: Average solar wind speed observed by the WIND satellite. During the first 100 days of the year both data sets are apparently correlated, their maxima exhibit a periodicity of about 27 days. Histogram binning used: 3 days.

the year 2002. In the upper panel 3 day average values of the solar wind speed measured with the WIND satellite are displayed. As can be seen, during the first 100 days of the year 2002 there is a good correlation between an observed high speed solar wind stream and the increase in the number of events or pulsation activity. The periodicity observed during this interval is therefore associated with the rotation period of the sun. After day of year 100 the correlation between both quantities is no longer apparent.

The distribution of the locations of the FLRs identified as the center of the latitudinal phase shift $x_{0}\left(\vartheta_{0}\right.$ in geomagnetic latitude) displays a broad peak around $67^{\circ}$ as shown in Fig. 6 . This reflects the locations of the stations of the CANOPUS array in the geomagnetic coordinate system. The preselection method is, of course, more sensitive for pulsations the amplitude of which maximizes at latitudes where the projected station density is highest. As the FLR location (the center of the phase shift area) coincides with the region of maximum amplitude, the distribution of the latitudinal FLR locations of the events should resemble to some extent the station configuration. The FLR locations obtained are therefore in most cases between $65^{\circ}$ and $73^{\circ}$ geomagnetic latitude, which is also consistent with the narrow bandwidth in frequency mentioned above.

The local time distributions of pulsation events of different frequencies are shown in Fig. 7. These distributions

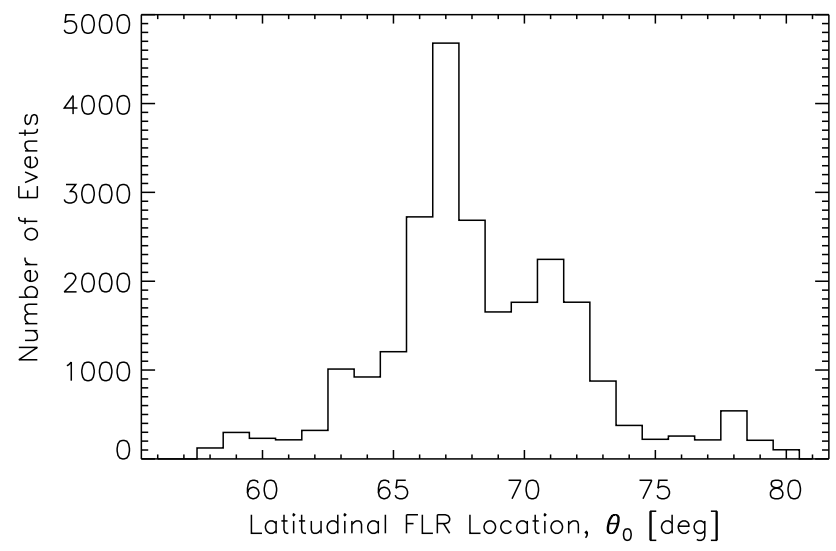

Fig. 6. Distribution of the latitudinal locations of the FLRs in geomagnetic coordinates. The distribution of the stations within the magnetometer array is reflected in the analysis. Binning used for the histogram: $1^{\circ}$.

have been normalized with respect to the total number of events in local time. Green and red bars show the differences to the mean percentage of occurrence of 100/24 (24 local time bins), which is indicated by the dotted lines. Taking into account the total event distribution in local time, pulsation events with relatively high frequencies (above $8 \mathrm{mHz}$ ) are more likely to occur around midnight or early morning. For pulsations of the lowest frequencies observed (between 2 and $3 \mathrm{mHz}$ ) this normalized maximum of occurrence is to be found at the morning and evening flank sectors, tending towards local noon for slightly higher frequencies (up to about $6 \mathrm{mHz}$ ). Taking into account that the location of most FLR events was determined to be within a relatively narrow geomagnetic latitude range (see Fig. 6) and assuming a single field line shell with its respective eigenfrequency to be responsible for large parts of the observed events, the distribution in Fig. 7 becomes explainable: Due to the parabolic shape of the magnetopause, the magnetosphere is more expanded at the flank sectors than at local noon. A single flux tube, the footpoint of which is located at a given geomagnetic latitude, is therefore expanded at the flanks and compressed at noon due to the direction of incidence of the solar wind. Its length varies with local time as well as its eigenfrequency. The resonance frequency of dayside FLR structures observed is, hence, highest at local noon, where the field lines are more compressed. The dependence of the occurrence probability for events with certain frequencies on local time is in excellent agreement with this picture, discussed in detail by Singer et al. (1981), who computed eigenperiods of field line shells in dependence on local time, geomagnetic latitude of the respective footpoints and average mass loading of the field lines. Experimental evidence for this effect has been presented more recently by Waters et al. (1995) using the cross-phase technique. 


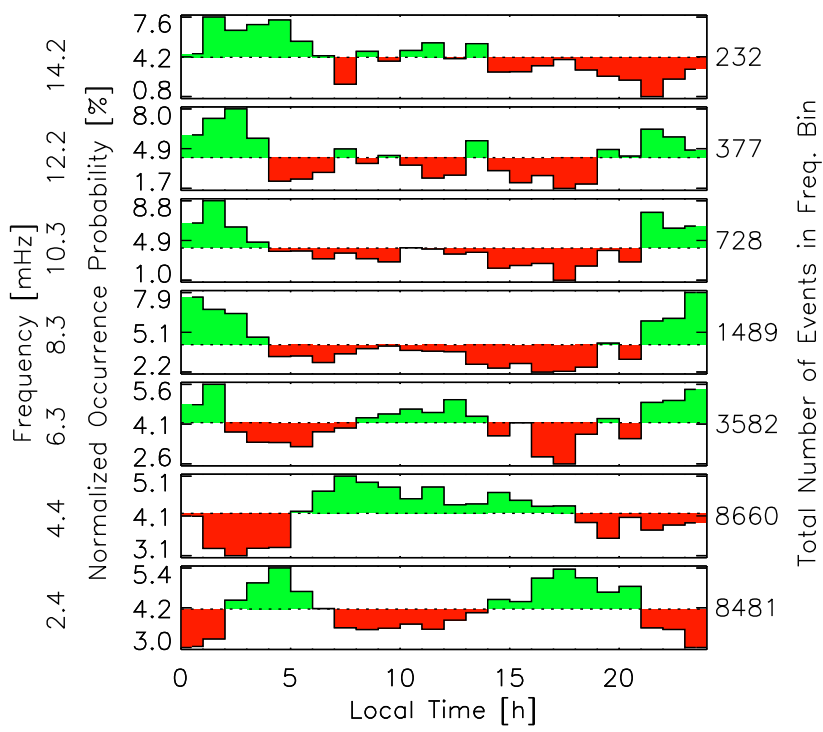

Fig. 7. Distributions of events of different frequency against local time normalized by the distribution of all events in local time. The percentage of occurrence of the events of each frequency bin is depicted as well as the total number of events (numbers at the right edge of the figure) to which this percentage corresponds. Red and green bars show the differences to the mean percentage of 100/24 indicated by the dotted lines. Local time binning used: one hour.

The interpretation of Fig. 8 instead may be more difficult. It shows distributions of events observed at different numbers of stations against local time. As in the previous Fig. 7, all distributions have been normalized with respect to the total number of events per local time bin. Events observed at a higher number of stations and therefore displaying a less localized amplitude structure are more likely to be found around local noon; in the midnight sector amplitude structures seem to be more localized instead. A possible interpretation can be done in terms of the latitudinal eigenfrequency gradient of the field line shells with footpoints located in the magnetometer array. The result could be explained assuming this gradient to increase from local noon until midnight. This would lead to a stronger localization of the pulsation amplitude over the array, resulting in less stations fulfilling the criterion for minimal power of pulsation and therefore in a decreasing average number of stations being involved in the FLRD analysis around local midnight.

Another possible explanation would be the narrowing of pulsation frequency bands with respect to the distance of observation from the subsolar point. Assuming that pulsations or oscillations of the dayside magnetopause contribute to the FLR structures observed, the magnetopause generated surface waves or associated compressible waves in the magnetosphere may become more narrow banded in frequency due to cavity or flank waveguide (Samson et al., 1992) eigenmodes. This would result in a narrowing of the radial amplitude dis-

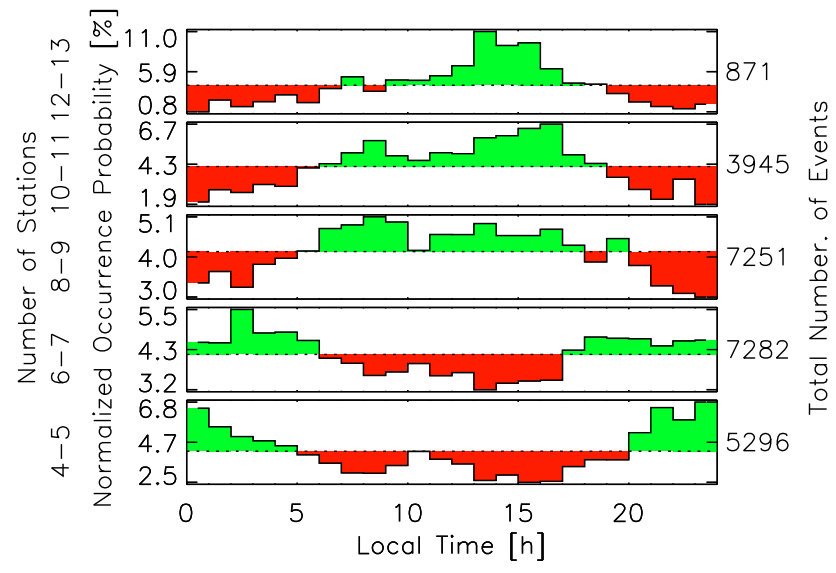

Fig. 8. Distribution of events seen with sufficient power at different numbers of stations against local time normalized by the distribution of all events in local time. The percentage of occurrence of events is depicted as well as the total number of events (number at the right edge of the figure) seen at different numbers of stations to which this percentage corresponds. Red and green bars show the differences to the mean percentage of $100 / 24$ indicated by the dotted lines. Local time binning: one hour.

tribution across the field line shells and to less stations observing significant pulsation power.

A third explanation is connected to the higher probability of occurrence of high frequency pulsation events at midnight which might suggest the prevalence of higher harmonics of standing Alfvén waves on nightside field lines. This would suggest a different wave generation mechanism to be dominant at these local times, such as an enhancement of waveparticle interactions. As higher harmonics are generally more localized in latitude, this would also explain why less stations are typically observing FLR wave structures around local midnight.

Figure 9 displays the percentaged distribution of the width of the phase shift $p$ in 5 bins (see figure and figure caption for details) around $p=0 \mathrm{~km}$ against local time. For FLRs this parameter is expected to be of the order of $100 \mathrm{~km}$ (e.g. Walker et al., 1979). The sign depends on the orientation of the radial gradient (on the ground: latitudinal gradient) of the field line eigenfrequency. When this gradient is directed earthward, as usual in the dayside magnetosphere outside the plasmapause, $p$ should display a small positive value. Since all the stations are located at high latitudes, the corresponding field line shells should be in most times outside the plasmasphere and we can conclude that the parameter $p$ should be in most cases greater than zero. As can be seen in the figure, there is a tendency for positive values of the parameter $p$ obtained from events observed in dayside local time sectors. This tendency is most pronounced for morning flank events that took place between 05:00 and 11:00 LT. For these times the percentage of $p>25 \mathrm{~km}$ events reaches or 


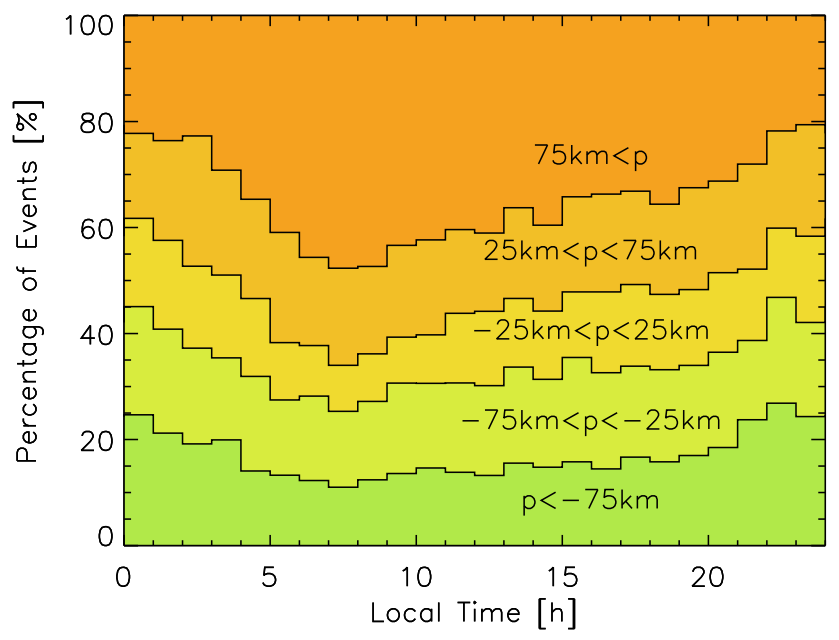

Fig. 9. Percentage of events showing a FLRD computed phase shift width $p$ in the intervals $p<-75 \mathrm{~km}$ (darker green), $-75 \mathrm{~km}<p<-25 \mathrm{~km}$ (light green), $-25 \mathrm{~km}<p<25 \mathrm{~km}$ (yellow), $25 \mathrm{~km}<p<75 \mathrm{~km}$ (light orange) and $75 \mathrm{~km}<p$ (darker orange) against local time. Binning of local time axis: one hour.

exceeds $60 \%$. Since independent tests of the FLRD method have proven the uncertainty in the determination of $p$ to be of the order of $50 \mathrm{~km}$, one can argue that some of the events showing a small negative $p$ may actually also belong to the positive bins. Although the percentage of positive phase shift width determinations decreases over local noon towards the dusk local time sectors, the global tendency remains. This is in concordance with the fact that the polarization should change over local noon as observed by Samson et al. (1971) and others. This change in polarization does only affect the azimuthal wavenumber $m$, which indeed changes sign as depicted in Fig. 10. It is also in agreement with the generally observed apparent poleward phase front motion in the resonance region of a FLR. The picture is different if we look to the distributions obtained for the midnight sectors (21:00 to 03:00 LT). Here positive and negative phase shift widths $p$ are in balance, even a slight prevalence of negative phase shift determinations is apparent. This may be also another indication for a different wave generation mechanism becoming dominant in the magnetosphere around local midnight. A negative $p$ could be in this case associated with an apparent equatorward phase front motion.

Figure 10 shows a two-dimensional histogram of events in dependence on the azimuthal wavenumber $m$ and local time. In the figure it is clearly visible that $m$ values in the negative range are predominant before local noon. The detected azimuthal wavenumbers concentrate between 0 and -5 and are therefore low, just as expected for toroidal mode FLR phase structures. In the post-noon sector $m$ values concentrate around 0 . Nevertheless, positive azimuthal wavenumbers below $m=5$ are overall predominant in this local time sector.

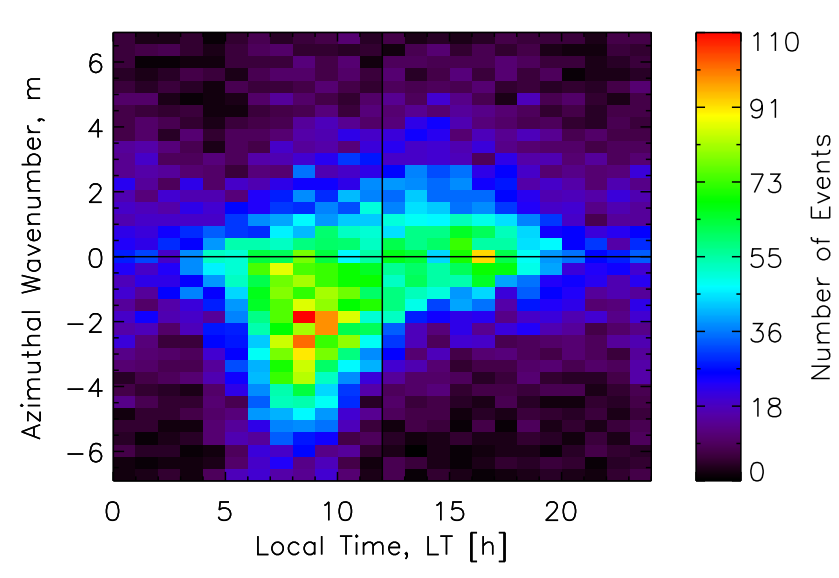

Fig. 10. Distribution of events with respect to the azimuthal wavenumber $m$ and local time of occurrence. Binning of $m$ axis: 0.37 .

These results are in good agreement with previous work presented by e.g. Olson and Rostoker (1978) or Chisham and Orr (1997) among others, who found a very similar distribution of azimuthal wavenumbers of Pc5 events against local time. This dawn-dusk asymmetry is generally attributed to a significant fraction of the FLR events being solar wind driven (e.g. by Kelvin-Helmholtz instability surface waves inducing inward propagating compressional disturbances) and thus showing a tailward motion of the phase structures observed on the ground. The change in sign across local noon is also in accordance with the predicted change in polarization observed and presented by Samson et al. (1971) in their pioneering work. The greater spread in $m$ for post-noon events around 0 may alternatively be the result of a larger fraction of non solar wind driven FLRs or coupling of cavity modes to standing Alfvén waves, that have also been shown to display low azimuthal wavenumbers (see Yeoman et al., 1990).

The frequency vs. azimuthal wavenumber distribution of the FLR events obtained here is very similar to the one obtained by Chisham and Orr (1997). The results are depicted separately for dawn (05:00-10:00 LT) and dusk (14:0019:00 LT) events in Figs. 11 and 12. In these histograms the distribution of events of each frequency bin with respect to the FLRD computed azimuthal wavenumbers $m$ is depicted. The result therefore emphasizes the azimuthal wavenumber most events were characterized with in each frequency bin. As can be seen in both figures, the azimuthal wavenumber $m$ of most events does not seem to be heavily dependent on the frequency. In the dawn sector azimuthal wavenumbers $m$ between 0 and -2 are favoured with large spreading in the range from -4 to 1 . In the dusk sector most events were characterized with low azimuthal wavenumbers between 0 and 1 .

In both cases no clear linear dependence of the azimuthal wavenumber $m$ on the frequency of the pulsation $f$ could be 


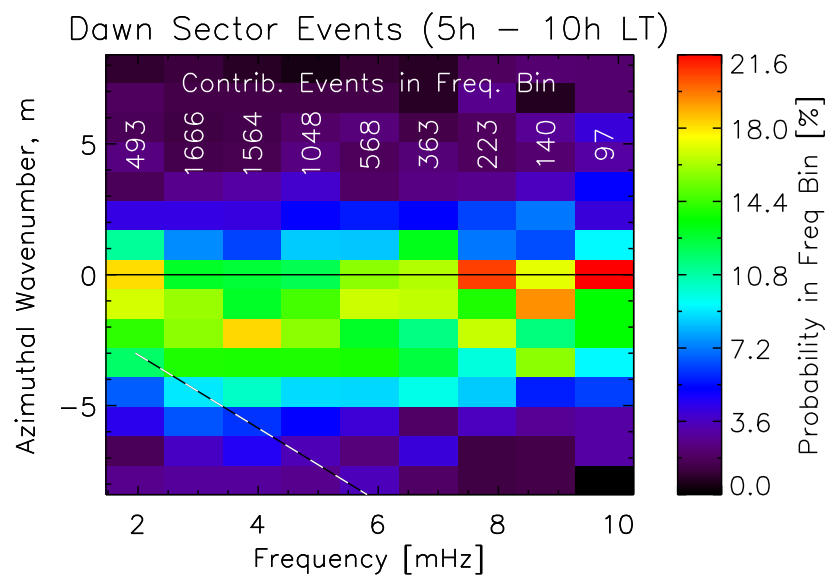

Fig. 11. Distribution of occurrence probability for dawn sector events (between 05:00 and 10:00 LT) within the frequency bins against the azimuthal wavenumber $m$. Binning of $m$ axis: 1.1. The number of events contributing to each frequency bin is shown in the upper part. The dashed line depicts the relation found by Olson and Rostoker (1978).

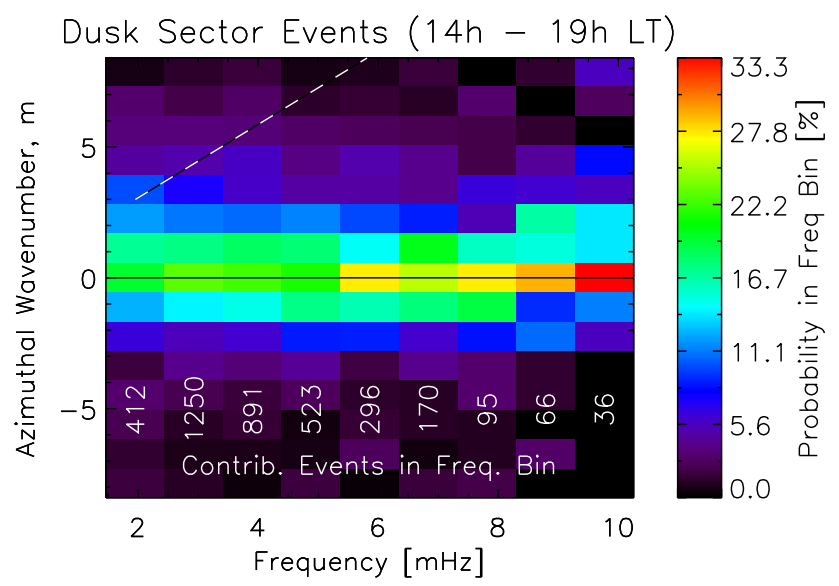

Fig. 12. Distribution of occurrence probability for dusk sector events (between 14:00 and 19:00 LT) within the frequency bins against the azimuthal wavenumber $m$. Binning of $m$ axis: 1.1. The number of events contributing to each frequency bin is shown in the lower part. The dashed line depicts the relation found by Olson and Rostoker (1978).

found. Therefore we have to point out the discrepancy with a relation found by Olson and Rostoker (1978):

$m=0.26+1.4 \cdot f$

where the frequency $f$ is given in units of $\mathrm{mHz}$. This linear relation suggesting a constant phase velocity in azimuthal direction is depicted with dashed lines in both Figs. 11 and 12. We conclude, as done before by Chisham and Orr (1997),

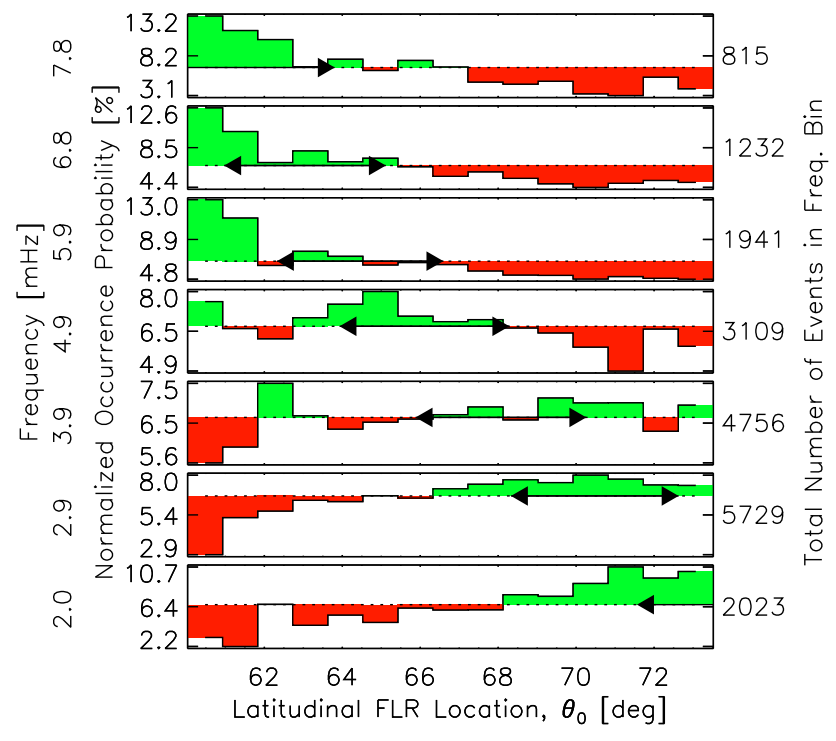

Fig. 13. Distribution of events of different frequency against the latitudinal FLR resonance location $\vartheta_{0}$ (computed with the FLRD) normalized by the distribution of all events in $\vartheta_{0}$. The percentage of occurrence of the events of each frequency bin is depicted as well as the corresponding total number of events to which this percentage is referred (numbers to the right of the figure). Red and green bars show the differences to the mean percentage indicated by the dotted lines. Arrow heads indicate latitudes computed for the corresponding frequencies using the relations found by Samson and Rostoker (1972) for the local time sectors 17:30-07:30 (lower latitude end) and 07:30-17:30 (upper latitude end).

that no clear dispersion relation $f(m)$ can be determined from the observations.

The distributions of events with different pulsation frequencies against the FLRD computed latitudinal FLR resonance locations are shown in Fig. 13. The distributions have been normalized with respect to the total number of events in each latitude bin. Red and green bars again depict the deviation from the mean percentage value of 100/15 (15 latitude bins). As expected, the normalized occurrence probability maximum decreases in FLR location latitude $\vartheta_{0}$ with increasing pulsation frequency. This is predicted as resonating field lines, whose footpoints are located at higher latitudes, are longer, since the respective field line shells are located at larger radial distance from Earth and therefore the corresponding eigenfrequecies decrease with increasing latitude of the footpoints. The FLR location is determined as the central latitude of the phase shift area of the observed pulsations, which in theory coincides with the point of maximum amplitude and hence with the point where the incoming compressible wave and the present field lines are in resonance. Thus Fig. 13 shows an average eigenfrequency profile.

A comparison of our results with relations found by Samson and Rostoker (1972) for the latitudinal dependence of the 
pulsation periods in the time sectors 17:30-07:30

$1 / f=T=-115+59.8 / \cos ^{2} \vartheta_{0}$

(lower latitude end arrow heads in Fig. 13) and 07:30-17:30

$1 / f=T=-58+37.4 / \cos ^{2} \vartheta_{0}$

(upper latitude end arrow heads in Fig. 13) shows that both results are in good agreement.

\section{Conclusions}

The presented statistical analysis of FLR-like wave structures observed using one year of ground based magnetic field data differs from previous work in the method used. The field line resonance detector has been proven to be an excellent tool for the characterization of FLR structures in narrow band ULF pulsations observed on the ground. Other automated systems of FLR activity recognition may only be applicable, when clear and single FLR structures are recognised. The classical methods check for criterions like phase shift over the amplitude maximum region. For this calculation a special array configuration in two straight lines of stations along a meridian and a parallel are necessary. The determination of the azimuthal wavenumber $m$ and the phase shift related parameters $\alpha$ and $p$ can only be computed by direct comparison of the relative phases of the pulsation signals observed at the different stations. If the overall measured phase shift does not match the established criterion (to be around $180^{\circ}$ ), the event is discarded.

The presented FLRD method instead works in a different way: Like the wave telescope, it is an estimator for the spectral energy density of the measured wave field in FLR characterizing parameter space. Therefore it is able to find hidden FLR phase structures because of the coherency across a large area covered by a couple of stations even when a clear signature is not apparent due to superposition! The only preselection that takes place is the frequency selection and determination of the duration of the pulsation events, common to all automated methods. In the presented work we filtered the FLR-like structures with the limitation of $\alpha$ being around $180^{\circ}$ in the domain scanned, which is part of the FLRD method. The results show that there are FLR-like structures hidden in one year of data, since the results are consistent with theory and previous work but with better statistics concerning the number of events that could be extracted with respect to previous automated FLR studies (e.g. Baker et al., 2003). The complete determination of parameters (even for multiple FLR events) is possible with this method. We have restricted ourselves to the strongest maxima found for each event to avoid a manual interpretation; nevertheless, complete determination is with this method no longer subject to a clear signal of a single FLR event.

Additionally, the FLRD method does only depend on the phase structure measured. The determination of all parameters is possible only with the phase. All the information subject to the amplitude distribution is still available for further examination. Particularly important is also the lack of dependence from a special positioning of the magnetometer array stations. As long as the FLR phase shift lies within the covered area, the locations of the stations may be completely arbitrary. However, the coordinate system in which the computations are performed has to be chosen carefully. The phase structures the FLRD searches for are fixed in the reference frame of the equirectangular projection. In particular the phase shift over the resonance region is assumed to take place only in latitudinal direction. Tests with synthetic FLR wave structure data have shown that a tilt of the assumed geomagnetic North Pole position and subsequently a wrong choice of the north-south direction for the transformation of the station positions into the equirectangular projection leads to a strong decrease in the spectral energy density of the maxima in parameter space indicating the parameters of the waves found, as well as to a slight deviation in their position. Consequently, a further improvement of the method might consist in the use of a more field line shell adapted coordinate system, for instance by connecting the equirectangular projection to the corrected geomagnetic coordinate system (CGM) instead of the simple polar geomagnetic coordinate system. Nevertheless, also results obtained by the classical methods of characterization are subject to the choice of the coordinate system. Thus we think that the spectral power density of the maxima could be used to adjust the reference coordinate system. Results can be discarded using this criterion, if the real alignment of the phase structure does not match the assumed one.

In the future the tool may be particularly helpful for single event characterization, when the event is not very clearly visible or when a superposition of two or more phase structures seems to be apparent (see Rae et al., 2007a). It should therefore be much more helpful in this task as the well known complex demodulation technique because of the impossibility to correctly determine all the FLR characterizing parameters with this traditional technique for several FLR structures at a time. Special adaptation to other situations, e.g. giant pulsations or determination of high $m$ structures in poloidal mode oscillations, seem to be possible and make the tool more valuable for future studies of ground and satellite based ULF pulsation measurements.

Acknowledgements. This work was financially supported by the German Ministerium für Wirtschaft und Technologie and the Deutsches Zentrum für Luft- und Raumfahrt under contract 50OC0103. CARISMA is operated by the University of Alberta, funded by the Canadian Space Agency. KHG is grateful for financial support through INTAS grant 05-1000008-7978. We acknowledge K. W. Ogilvie and A. J. Lazarus for the WIND satellite based solar wind data.

Topical Editor I. A. Daglis thanks two anonymous referees for their help in evaluating this paper. 


\section{References}

Allan, W., White, S. P., and Poulter, E. M.: Impulse-excited hydromagnetic cavity and field-line resonances in the magnetosphere, Planet. Space Sci., 34, 371-385, doi:10.1016/0032-0633(86) 90144-3, 1986.

Baker, G. J., Donovan, E. F., and Jackel, B. J.: A comprehensive survey of auroral latitude Pc5 pulsation characteristics, J. Geophys. Res., 108, 1384-1397, doi:10.1029/2002JA009801, 2003.

Beamish, D., Hanson, H. W., and Webb, D. C.: Complex demodulation applied to $\mathrm{Pi} 2$ geomagnetic pulsations, Geophys. J. Int., 58, 471-493, doi:10.1111/j.1365-246X.1979.tb01035.x, 1979.

Capon, J.: High resolution frequency-wavenumber spectrum analysis, 57, 1408-1418, 1969.

Capon, J., Greenfield, R. J., and Kolker, R. J.: Multidimensional maximum-likelihood processing of a large aperture seismic array, P. IEEE, 55, 192-213, 1967.

Chen, L. and Hasegawa, A.: A theory of longperiod magnetic pulsations. 1. Steady state excitation of field line resonance, J. Geophys. Res., 79, 1024-1032, 1974.

Chisham, G. and Orr, D.: A statistical study of the local time asymmetry of Pc 5 ULF wave characteristics observed at midlatitudes by SAMNET, J. Geophys. Res., 102, 24 339-24350, doi:10.1029/97JA01801, 1997.

Constantinescu, O. D., Glassmeier, K.-H., Décréau, P. M. E., Fränz, M., and Fornaçon, K.-H.: Low frequency wave sources in the outer magnetosphere, magnetosheath, and near Earth solar wind, Ann. Geophys., 25, 2217-2228, 2007,

http://www.ann-geophys.net/25/2217/2007/.

Dungey, J. W.: Electrodynamics of the outer atmosphere, vol. 69 of Ionosphere Research Laboratory Science Report, Pennsylvania State University, Pennsylvania, 1954.

Engebretson, M., Glassmeier, K.-H., Stellmacher, M., Hughes, W. J., and Lühr, H.: The dependence of high-latitude Pc5 wave power on solar wind velocity and on the phase of high-speed solar wind streams, J. Geophys. Res., 103, 26 271-26 283, 1998.

Fenrich, F. R., Samson, J. C., Sofko, G., and Greenwald, R. A.: ULF high- and low-m field line resonances observed with the Super Dual Auroral Radar Network, J. Geophys. Res., 100, 21 53521 548, doi:10.1029/95JA02024, 1995.

Fujita, S., Glassmeier, K. H., and Kamide, K.: MHD waves generated by the Kelvin-Helmholtz instability in a nonuniform magnetosphere, J. Geophys. Res., 101, 27 317-27 326, doi:10.1029/ 96JA02676, 1996.

Glassmeier, K.-H.: Reconstruction of the ionospheric influence on ground-based observations of a short-duration ULF pulsation event, Planet. Space Sci., 36, 801-817, doi:10.1016/ 0032-0633(88)90086-4, 1988.

Glassmeier, K.-H. and Stellmacher, M.: Concerning the local time asymmetry of Pc5 wave power at the ground and field line resonance widths, J. Geophys. Res., 105, 18 847-18 856, doi: 10.1029/2000JA900037, 2000.

Glassmeier, K. H., Lester, M., Mier-Jedrzejowicz, W. A. C., Green, C. A., Rostoker, G., Orr, D., Wedeken, U., Junginger, H., and Amata, E.: Pc5 pulsations and their possible source mechanisms - A case study, J. Geophys. Res., 55, 108-119, 1984.

Glassmeier, K.-H., Othmer, C., Cramm, R., Stellmacher, M., and Engebretson, M.: Magnetospheric Field Line Resonances: A Comparative Planetology Approach, Surv. Geophys., 20, 61$109,1999$.
Glassmeier, K.-H., Motschmann, U., Dunlop, M., Balogh, A., Acuña, M. H., Carr, C., Musmann, G., Fornaçon, K.-H., Schweda, K., Vogt, J., Georgescu, E., and Buchert, S.: Cluster as a wave telescope - first results from the fluxgate magnetometer, Ann. Geophys., 19, 1439-1447, 2001,

http://www.ann-geophys.net/19/1439/2001/.

Green, C. A.: Continuous magnetic pulsations on the IGS array of magnetometers, J. Atmos. Terr. Phys., 43, 883-898, 1981.

Greenwald, R. A. and Walker, A. D. M.: Energetics of long period resonant hydromagnetic waves, Geophys. Res. Lett., 7, 745-748, 1980.

Hughes, W. J.: The effect of the atmosphere and ionosphere on long period magnetospheric micropulsations, Planet. Space Sci., 22, 1157-1172, doi:10.1016/0032-0633(74)90001-4, 1974.

Mann, I. R., Wright, A. N., Mills, K. J., and Nakariakov, V. M.: Excitation of magnetospheric waveguide modes by magnetosheath flows, J. Geophys. Res., 104, 333-354, doi:10.1029/ 1998JA900026, 1999.

Maus, S., MacMillan, S., Chernova, T., Choi, S., Dater, D., Golovkov, V., Lesur, V., Lowes, F., Lühr, H., Mai, W., McLean, S., Olsen, N., Rother, M., Sabaka, T., Thomson, A., Zvereva, T., and International Association of Geomagnetism, Aeronomy (IAGA), Division V, Working Group VMOD: The 10th generation international geomagnetic reference field, Phys. Earth Planet. In., 151, 320-322, doi:10.1016/j.pepi.2005.03.006, 2005.

Motschmann, U., Woodward, T. I., Glassmeier, K. H., Southwood, D. J., and Pinçon, J. L.: Wavelength and direction filtering by magnetic measurements at satellite arrays: Generalized minimum variance analysis, J. Geophys. Res., 101, 4961-4966, doi: 10.1029/95JA03471, 1996.

Narita, Y., Glassmeier, K.-H., Schäfer, S., Motschmann, U., Sauer, K., Dandouras, I., Fornaçon, K.-H., Georgescu, E., and Rème, H.: Dispersion analysis of ULF waves in the foreshock using cluster data and the wave telescope technique, Geophys. Res. Lett., 30, 1710-1713, doi:10.1029/2003GL017432, 2003.

Olson, J. V. and Rostoker, G.: Longitudinal phase variations of Pc 4-5 micropulsations, J. Geophys. Res., 83, 2481-2488, 1978.

Pillai, S. U.: Array signal processing, chapter 2, p. 8-107, SpringerVerlag, New York, 1989.

Pinçon, J. L. and Lefeuvre, F.: Local characterization of homogeneous turbulence in a space plasma from simultaneous measurements of field components at several points in space, J. Geophys Res., 96, 1789-1802, 1991.

Rae, I. J., Donovan, E. F., Mann, I. R., Fenrich, F. R., Watt, C. E. J., Milling, D. K., Lester, M., Lavraud, B., Wild, J. A., Singer, H. J., Rème, H., and Balogh, A.: Evolution and characteristics of global Pc5 ULF waves during a high solar wind speed interval, J. Geophys. Res., 110, 12211-12 226, doi:10. 1029/2005JA011007, 2005.

Rae, I. J., Mann, I. R., Dent, Z. C., Milling, D. K., Donovan, E. F., and Spanswick, E.: Multiple field line resonances: Optical, magnetic and absorption signatures, Planet. Space Sci., 55, 701-713, doi:10.1016/j.pss.2006.02.009, 2007a.

Rae, I. J., Watt, C. E. J., Fenrich, F. R., Mann, I. R., Ozeke, L. G., and Kale, A.: Energy deposition in the ionosphere through a global field line resonance, Ann. Geophys., 25, 2529-2539, 2007b, http://www.ann-geophys.net/25/2529/2007/.

Rostoker, G., Samson, J. C., Creutzberg, F., Hughes, T. J., McDiarmid, D. R., McNamara, A. G., Jones, A. V., Wallis, D. D., 
and Cogger, L. L.: Canopus - A ground-based instrument array for remote sensing the high latitude ionosphere during the ISTP/GGS program, Space Sci. Rev., 71, 743-760, doi:10.1007/ BF00751349, 1995.

Samson, J. C. and Rostoker, G.: Latitude-Dependent Characteristics of High-Latitude Pc 4 and Pc 5 Micropulsations, J. Geophys. Res., 77, 6133-6144, doi:10.1029/JA077i031p06133, 1972.

Samson, J. C., Jacobs, J. A., and Rostoker, G.: Latitude-Dependent Characteristics of Long-Period Geomagnetic Micropulsations, J. Geophys. Res., 76, 3675-3683, doi:10.1029/JA076i016p03675, 1971.

Samson, J. C., Harrold, B. G., Ruohoniemi, J. M., Greenwald, R. A., and Walker, A. D. M.: Field line resonances associated with MHD waveguides in the magnetosphere, Geophys. Res. Lett., 19, 441-444, 1992.

Singer, H. J., Southwood, D. J., Walker, R. J., and Kivelson, M. G.: Alfven wave resonances in a realistic magnetospheric magnetic field geometry, J. Geophys. Res., 86, 4589-4596, 1981.

Southwood, D. J.: Some features of field line resonances in the magnetosphere, Planet. Space Sci., 22, 483-491, doi:10.1016/ 0032-0633(74)90078-6, 1974.
Tamao, T.: Transmission and coupling resonance of hydrodynamic disturbances in the non-uniform Earth's magnetosphere, Sci. Rep. Tohoku Univ., Fifth Ser., 17, 43-72, 1965.

Walker, A. D. M., Greenwald, R. A., Stuart, W. F., and Green, C. A.: STARE auroral radar observations of Pc 5 geomagnetic pulsations, J. Geophys. Res., 84, 3373-3388, 1979.

Waters, C. L., Samson, J. C., and Donovan, E. F.: The temporal variation of the frequency of high latitude field line resonances, J. Geophys. Res., 100, 7987-7996, 1995.

Yeoman, T. K., Lester, M., Orr, D., and Lühr, H.: Ionospheric boundary conditions of hydromagnetic waves: The dependence on azimuthal wavenumber and a case study, Planet. Space Sci., 38, 1315-1325, doi:10.1016/0032-0633(90)90134-C, 1990.

Ziesolleck, C. W. S. and McDiarmid, D. R.: Statistical survey of auroral latitude Pc 5 spectral and polarization characteristics, J. Geophys. Res., 100, 19299-19312, doi:10.1029/95JA00434, 1995. 